\title{
Tertiary trisomics in the garden pea as a model of $B$ chromosome evolution in plants
}

\author{
VA Berdnikov, FL Gorel, OE Kosterin and VS Bogdanova \\ Institute of Cytology E Genetics, Siberian Division of Russian Academy of Sciences, Acad. Lavrentiev Avenue 10, Novosibirsk 630090, \\ Russia
}

It is hypothesized that, in plants, genetically empty B chromosomes may originate from the extra chromosome (E) of tertiary trisomics if (i) the region of basic chromosomes homologous to the $\mathrm{E}$ (H-region) harbors a sporophytic lethal covered by the wild-type allele in $\mathrm{E}$, and (ii) crossing-over between $\mathrm{E}$ and the $\mathrm{H}$-region is suppressed. Under these conditions, most loss-of-function mutations occurring in the $\mathrm{H}$-region are deleterious for haploid gametophytes, whereas those occurring in $E$ are neutral or advantageous for hyperploid $(n+1)$ gametophytes. As a result, natural selection at the gametophyte level can lead to the degeneration of $\mathrm{E}$, leaving the $\mathrm{H}$-region intact. Using Hammarlund translocation T(3-6)a, we synthesized two trisomic lines of the garden pea (Pisum sativum L.), where $\mathrm{E}$ was composed of the short arms of chromosomes 3 and 6 and the H-region carried recessive markers. In the trisomic line TRIS, we found few crossovers between $\mathrm{E}$ and the $\mathrm{H}$-region. In the trisomic line TRUST, obtained after a change of basic chromosome constitution, recombination in this region was completely suppressed. After induction in the H-region of TRUST of a recessive sporophytic mutation $r m v$, two 15-chromosome lines of stable trisomics were established. One of them passed 11 generations, having produced more than 6000 individuals, all of them trisomic, and $\mathrm{E}$ remained present as a single element with no pairing partners. No tetrasomics were dete-cted in these lines. If such trisomics occurred in nature, their extra chromosomes are likely to become a B chromosome.

Heredity (2003) 91, 577-583, advance online publication, 17 September 2003; doi:10.1038/sj.hdy.6800357

Keywords: B chromosomes; plants; tertiary trisomy; translocations; gametophytic lethals; Pisum sativum L.

\section{Introduction}

In about $15 \%$ of the animal and plant species, the karyotype of some individuals contains one or more chromosomes in excess of the basic set (Bukeboom, 1994). The properties of these supernumerary, or B, chromosomes have been comprehensively reviewed by Jones and Rees (1982). These chromosomes generally do not carry genes with a major effect. The presence of one B chromosome exerts little effect on the organism, although their accumulation in the genome can result in a noticeable decline of viability, fertility and developmental rate. How these dispensable genomic elements arise remains unresolved, although their origin from the regular chromosomes seems to be most likely (Jones and Rees, 1982).

Sex chromosomes of animals resemble Bs in many respects. For example, the $\mathrm{Y}$ chromosome of Drosophila melanogaster has only a dozen genes, mostly responsible for male fertility (Adams et al, 2000). The presence of one $\mathrm{Y}$ chromosome in the female and two Ys in the male has no phenotypic effect, but higher numbers of Ys result in some reduction of fitness (Ashburner, 1989). Such properties of sex chromosomes can allow their practically instantaneous transformation into Bs (Green et al,

Correspondence: VA Berdnikov, Institute of Cytology \& Genetics, Siberian Division of Russian Academy of Sciences, Acad. Lavrentiev Avenue 10, Novosibirsk 630090, Russia. E-mail: berdnik@bionet.nsc.ru

Received: 20 June 2002; revised: 24 May 2003; accepted: 10 July 2003
1993; Colombo and Remis, 1997). However, in plants, generally lacking sex chromosomes, the origin of genetically empty chromosomes appears more enigmatic. It is widely accepted that B chromosomes are derived from some regular chromosomes or from their fragments (for a review, see Jones and Rees, 1982), and the plant genome containing a future B must pass through the stage of trisomy.

However, it remains unclear as to how this transition occurs, taking into account the genic imbalance. There are two ways to overcome such genic imbalance: (i) suppression of gene activity on the extra chromosome converting chromatin into transcriptionally silent state (Jones and Rees, 1982; Green, 1990), as occurs in mammalian $X$ chromosomes, (ii) accumulation of deleterious mutations in the extra chromosome leaving the homologous region of the genome intact. This region is further referred to as the 'H-region'.

To date, there is little information on how silencing of entire chromosome takes place in plants. For the second of the two alternatives to occur, two conditions must be satisfied: (i) the same extra chromosome must persist in a population for many generations, in spite of strong selection in favor of euploids and a high probability of loss at meiosis; and (ii) there must exist a mechanism removing deleterious mutations from all genome regions except the extra chromosome. Below, we show that both conditions can be satisfied for tertiary trisomics.

In primary trisomics, all three homologous chromosomes are the same and it is hard to imagine how one of them can be separated and evolve into a B chromosome. 
In addition, the rate of arisal of primary trisomics is too low to explain a high frequency of species with supernumeraries. Tertiary trisomics, with the extra chromosome composed of segments of two nonhomologous chromosomes of the basic set, seem better suited for B chromosome formation. They regularly appear in the progenies of heterozygotes for translocations (eg, Ramage, 1960), which are known to be widespread in plant populations (Lewis, 1951; James, 1965; Wedberg et al, 1968). Moreover, it is well known that in wild populations, plants heterozygous for a translocation may contain sporophytic lethals near the breakpoints (Cleland, 1962; James, 1965). If recombination between the lethal and the breakpoint is suppressed, the progeny of a self-pollinated trisomic will consist predominantly of trisomics with the parental karyotype, because disomics die, and tetrasomics have a poor chance to survive due to severe genic imbalance (Figure 1). Each of the surviving trisomics may become a founder of a population consisting entirely of trisomics. In a population of 'stable' trisomics, natural selection will fix deleterious mutations bringing the trisomic genome closer to the more advantageous diploid state. However, this diploidization of trisomics would lead to inactivation of genes residing not only in the extra chromosome but also in the $\mathrm{H}$ region of basic chromosomes.

It is important to note that the life cycle of angiosperms includes a haploid phase, and that a large proportion of genes expressed in the sporophyte may be active in the male gametophyte as well (Mascarenhas, 1989; Ottaviano and Mulcahy, 1989). Thus, the majority of sporophytic

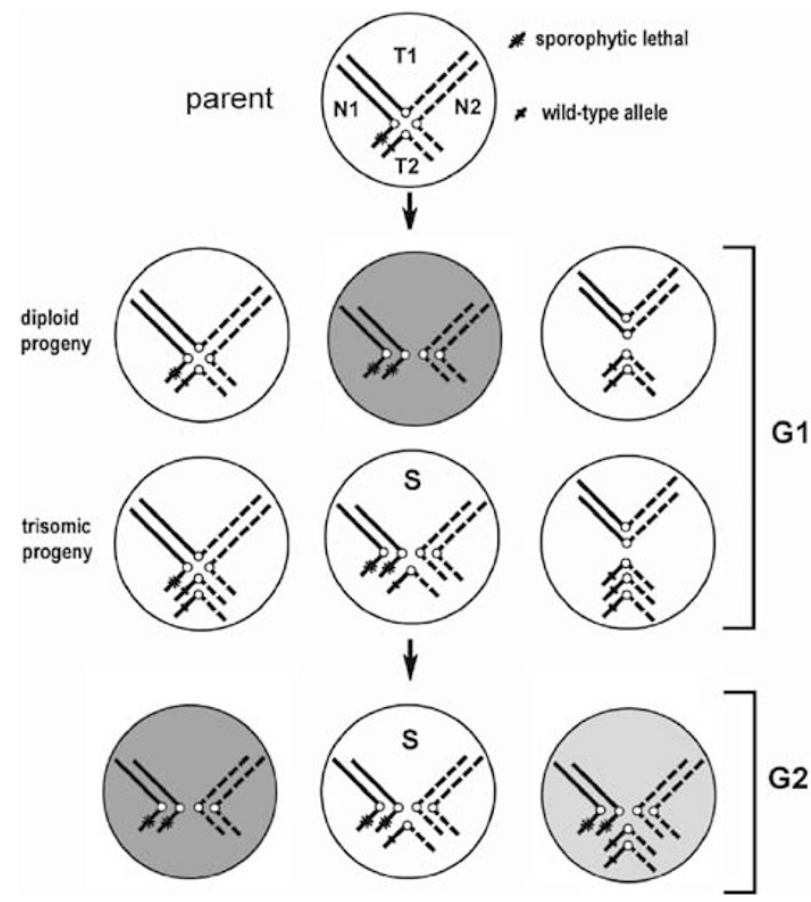

Figure 1 Appearance of stable trisomics in the progeny of a selfpollinated translocation heterozygote. Only trisomics with an extra chromosome composed of short arms of basic chromosomes are shown. N1, N2 - chromosomes with a normal gene order; T1 - large translocated chromosome; T2 - small translocated chromosome. Inviable diploids are given in dark gray, poorly viable tetrasomics in light gray. S - stable trisomics. G1, G2 - first and second generations. a
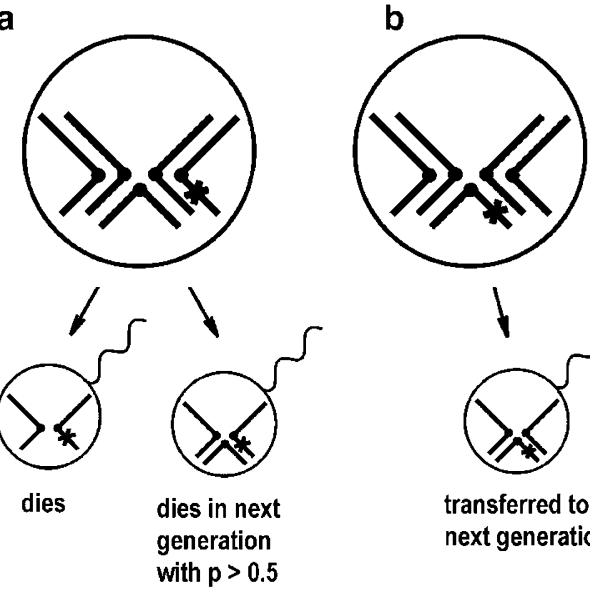

gametophytic lethal

SPOROPHYTES

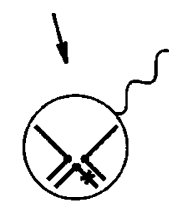

GAMETOPHYTES

transferred to

next generation

Figure 2 Fate of a gametophytic lethal in a population of tertiary trisomics. (a) lethal in the H-region; (b) lethal in the extra chromosome.

loss-of-function mutations or deletions may have a negative effect on the viability and competitive ability of the male gametophytes. Let us trace the fate of a recessive gametophytic lethal in the H-region of a tertiary trisomic (Figure 2). Gametophytes may be of two kinds - ordinary haploid gametophytes and hyperploid $(n+1)$ ones carrying the extra chromosome. The haploid gametophyte cannot transfer a lethal to the next generation, while the $n+1$ gametophyte can, since in this case the lethal is covered by its wild-type allele in the extra chromosome. Nevertheless, in the next generation, the lethal, with a probability $>0.5$, will be included into a haploid gametophyte and be lost. Thus, a gametophytic lethal in the H-region will be removed from the population of stable trisomics over several generations.

In contrast, any gametophytic lethal occurring in the extra chromosome will constantly be covered by a wildtype allele of the H-region, and nothing prevents it from persistence in the extra chromosomes of trisomic offspring. Thus, in a population of stable trisomics, the accumulation of gametophytic lethals will be biased to the extra chromosome. To guarantee such a different fate of initially equivalent genomic elements, recombination between the future $\mathrm{B}$ and the H-region must be suppressed so that inactivated genes in the extra chromosome could not be replaced by wild-type alleles. Thus, both conditions needed for B chromosome formation, a long life of the extra chromosome and its preferential degradation, can be satisfied if this chromosome is isolated from recombination. This is not improbable, since, at meiotic metaphase I, the extra chromosome of some tertiary trisomics is often detected as a univalent (Vasek, 1961; Khush and Rick, 1967).

In this work, we have made an attempt to reproduce the initial stages of $\mathrm{B}$ chromosome evolution experimentally. As a model, we have chosen the garden pea (Pisum sativum L.), which is relatively well studied genetically. The heterozygotes for Hammarlund translocation, T(36)a, regularly produced tertiary trisomics with a small interchange chromosome $3^{6}$, composed of the short arms of chromosomes 3 and 6 (Sutton, 1939; Pellew, 1940; Lamm, 1986). This extra chromosome may be regarded 
as a candidate for incipient B. Having introduced recessive markers into the $\mathrm{H}$-region, we found that recombination on the extra chromosome was substantially suppressed. After the induction of a sporophytic lethal in the H-region, two stable trisomic lines were established with the extra chromosomes prone to degradation directed by natural selection acting both on the gametophyte and sporophyte levels.

\section{Materials and methods}

\section{Genetic markers used}

a, long arm of chromosome 6 (Linkage Group II) - no anthocyanine coloration;

$c r d^{w h}$, short arm of chromosome 6 (Linkage Group II) very long rachis with reduced leaflets and lateral tendrils;

gp, long arm of chromosome 3 (Linkage Group V) yellow pods;

cri, proximal region of chromosome 3 short arm (Linkage Group V) - leaf petioles very short, leaflet margins curled;

$U^{\text {st }}$, distal region of chromosome 3 short arm (Linkage Group V) - anthocyanine stripes on seed testa, the seeds of $u$-plants lack the stripes.

\section{Diploid lines}

WL1476 ( = Hammarlund's K-line = Lamm's L84) homozygous for translocation, T(3-6)a, involving chromosomes 3 and 6, corresponding to Linkage Groups V and II, respectively; from the germplasm collection at Weibullsholm, Landscrona (Sweden).

WL1238 - a multimarker testerline from the germplasm collection at Weibullsholm, Landscrona (Sweden).

SG - a stock obtained in our laboratory on the basis of the accessions VIR6135 (Greece), VIR320 (P.s. syriacum, Palestine) and the original line Sprint- 1 .

SGE182 $(A$, cri $u)$, - a line carrying the mutation cri induced by EMS in the stock SG.

WHAF $\left(a c r d^{w h}, u\right)$ - derived from the mutant $c r d^{w h}$ induced by EMS in the stock SG, VIR5538 (Daghestan), WL1238, and the cv. Frontier, courtesy of Dr E Gritton.

Yellow Crispa $(A, g p$ cri $u)$ derived from $\mathrm{F} 2$ of the cross WL1238 × SGE182.

\section{Trisomic lines}

The founder of the line TRIS was a trisomic plant with $g p$ Cri phenotype found in the progeny of the testcross $($ WL1476 $\times$ Yellow Crispa $) \times$ Yellow Crispa. Trisomic plants can be distinguished from diploids by enlarged bracts (Sutton, 1939), an increased number of flowers per inflorescence and slightly undulated margins of stipules and leaflets (Berdnikov et al, 1993), and the mean number of seeds per pod (3.5) is reduced compared to ordinary diploids. The founder of the line TRUST was derived as a single F13 plant from a cross of a TRIS plant with a diploid plant cri $U^{\text {st }}$ from F2 of the cross WL1238 $\times$ SGE182. The founder of the line TRUST-R was an M5 plant after EMS treatment of the line TRUST. (seeds of the TRUST line were left to imbibe $0.1 \%$ EMS during $18 \mathrm{~h}$ and immediately sown in the greenhouse). The founder of the line TRAC was a trisomic plant with phenotype $(a \mathrm{Crd})$ found in F2 from the cross TRUST$\mathrm{R} \times$ WHAF. Both stable trisomic lines have reduced number of seeds (1.5-2) per pod.

\section{Cytological preparations}

For analysis of metaphase I of pollen mother cells, flower buds (1-2 mm) were fixed and stored in Carnoy's solution (ethanol-acetic acid (3:1)). The preparations were stained with $2 \%$ acetocarmine and squashed in the same solution.

For analysis of mitotic chromosomes, root tips (60$80 \mathrm{~mm}$ in length) were excised and kept in a saturated solution of 1 -bromonaphtalene at $4{ }^{\circ} \mathrm{C}$ for $12 \mathrm{~h}$, fixed in Carnoy's solution, hydrolyzed in $0.2 \mathrm{M} \mathrm{HCl}$ at $60^{\circ} \mathrm{C}$ for 20-30 s, washed in distilled water, stained with heated $2 \%$ acetocarmine and squashed in $45 \%$ acetic acid.

The estimates of pollen fertility were based on the frequency of pollen grains stained with $2 \%$ acetocarmine in acetic acid (45\%). Inviable grains did not stain red, were shrunken and devoid of cytoplasm.

\section{Linkage estimation}

The linkage between cri and the lethal $r m v$ was estimated by the maximum likelihood method (Kosterin et al, 1999).

\section{Plant cultivation}

Plants were grown in the greenhouse of the Institute of Cytology \& Genetics, Novosibirsk, in hydroponic vermiculite/ceramsite $(2: 1, \mathrm{v} / \mathrm{v})$ beds fed by standard Knop nutrient solution four times a day. They were illuminated by $8 \mathrm{~h}$ daylight $/ 16 \mathrm{~h}$ incandescent light of 10000 $120001 x$ intensity. The field trials were carried out in the environs of Novosibirsk Academy Town.

\section{Results}

\section{Recombination in the extra chromosome of tertiary trisomics in pea}

In 1992, we obtained a trisomic plant in which both chromosomes 3 (Linkage Group V) carried the recessive mutation crispa (cri) in the short arms, while the small extra chromosome $3^{6}$, composed of the short arms of chromosomes 3 and 6, had the wild-type allele Cri situated near the translocation breakpoint (Berdnikov et al, 1993). The mutation cri sharply distorts the foliage. In the progeny of the self-pollinated trisomics, diploid cri-plants can be easily distinguished from trisomic Criplants with genotype cri/cri/(Cri) (Figure 3). Hereafter, in the designation of trisomic genotype, genes of the extra chromosome are given in parentheses. The region of diploid genome homologous to the extra chromosome, which is the short arms of chromosomes 3 and 6, will be referred to as the 'H-region'. One trisomic plant became a founder of the line TRIS. In this line, the proportion of trisomics among selfed progenies ranged from 10 to $30 \%$ with the mean value of $18.7 \%$ (Table 1 ). This allowed maintenance of the line by self-pollination. Among 1039 diploids of the line TRIS, four exceptional plants with the phenotype $\mathrm{Cri}$ were registered. These must have appeared due to recombination between cri and the translocation breakpoint.

To introduce additional markers into the H-region, we crossed a trisomic of the line TRIS (cri u/cri u/(Cri u)) used as a pollen parent with a diploid plant (cri $\left.U^{s t} / c r i U^{s t}\right)$. The locus $u$ resides in the telomeric region of the short arm of chromosome 3 , the dominant allele $U^{s t}$ conditions violet stripes on the seed testa. One trisomic F1 plant became a founder of the trisomic line called TRUST. 


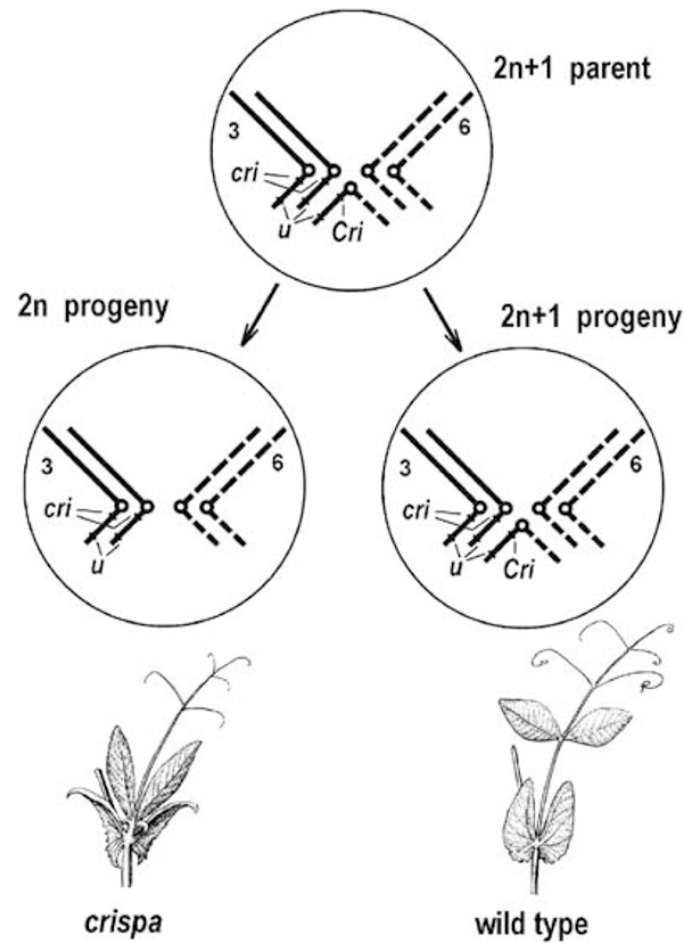

Figure 3 Progeny types of self-pollinated trisomics of the line TRIS. Numbers stand for chromosomes 3 and 6 (Linkage Groups V and II).

Table 1 Transmission of the extra chromosome in self-pollinated trisomics and in crosses of trisomics ( $q$ and $\hat{\delta})$ with diploids $(2 n)$

\begin{tabular}{lcccc}
\hline Trisomic & $\begin{array}{c}\text { Type of } \\
\text { pollination }\end{array}$ & \multicolumn{3}{c}{ Progeny } \\
\cline { 3 - 5 } & & $\begin{array}{c}\text { Number of } \\
\text { trisomics }\end{array}$ & \% trisomics & $\begin{array}{c}\text { Total } \\
\text { number }\end{array}$ \\
\hline TRUST & selfing & 2284 & 27.0 & 8459 \\
& $\times 2 n$ & 98 & 25.5 & 383 \\
TRUST-R & $2 n \times$ & 121 & 12.3 & 983 \\
& $\times 2 n$ & 42 & 34.4 & 122 \\
TRIS & $2 n \times$ & 31 & 17.6 & 176 \\
& selfing & 239 & 18.7 & 1278 \\
\hline
\end{tabular}

As a diploid parent, the line Yellow Crispa was used.

After 13 generations of isogenization by selfing of one randomly chosen trisomic in each generation, one vigorous trisomic plant was propagated to give thousands of trisomics. In the progenies of self-pollinated trisomics of the TRUST line, approximately $73 \%$ of the plants were diploids and the rest were trisomics (Table 1). All the plants had the $U^{\text {st }}$ phenotype. The most striking feature of the TRUST line was the complete absence of plants with the Cri-phenotype among 6175 diploids in the progenies of trisomics (Table 1). This implies a complete absence of crossing-over between cri and the breakpoint of translocation.

The absence of plants with the recessive phenotype $u$ in the TRUST line can indicate that all three copies of the locus $u$ would be represented by the dominant allele $U^{s t}$. To test it, we crossed a trisomic TRUST plant taken as a male with the diploid line Yellow Crispa (cri $u$ ) and analyzed phenotypic segregation in the progenies of
Table 2 Segregation ratios in disomic and trisomic progeny of selfpollinated trisomics obtained from the cross TRUST $\times$ Yellow Crispa (cri $u$ ).

\begin{tabular}{|c|c|c|c|c|c|}
\hline \multirow[t]{2}{*}{ F2 group } & \multirow[t]{2}{*}{ Total } & \multicolumn{3}{|c|}{ Phenotype } & \multirow{2}{*}{$\frac{\chi^{2}}{3: 1}$} \\
\hline & & $u$ & Ust & $\% u$ & \\
\hline Disomics & 86 & 23 & 63 & 26.7 & $0.14^{\mathrm{a}}$ \\
\hline Trisomics & 55 & 13 & 41 & 23.6 & $0.04^{\mathrm{a}}$ \\
\hline
\end{tabular}

selfed F1 trisomics. In F2, trisomics and diploids were analyzed separately. It was found (Table 2) that in both F2 groups, the proportion of $u$-plants did not differ significantly from $1 / 4$. Such a result demonstrated that trisomics of the line TRUST had the genotype $U^{s t} / U^{s t} /(u)$. If their genotype were $U^{s t} / U^{s t} /\left(U^{s t}\right)$ and that of $\mathrm{F} 1$ trisomics $-u / U^{s t} /\left(U^{s t}\right)$, practically all the trisomics of F2 would have the dominant $U^{s t}$ phenotype. Even admitting free recombination between loci $c r i$ and $u$, the proportion of trisomics with the genotype $u / u /(u)$ would not exceed $1 / 27$. Hence, the extra chromosome in the TRUST line, in spite of 13 generations of isogenization, still retains its initial allele $u$. (A similar approach for identifying the genotype of trisomics was applied by Khush and Rick, 1967 in tomato.) These results allow us to conclude that at least one arm (homologous to chromosome 3) of the extra chromosome is excluded from recombination in trisomics of the line TRUST.

Another interesting feature of the lines TRIS and TRUST was the complete absence of tetrasomics in the progenies of trisomics. All Cri-plants had quite uniform habitus, and after self-pollination produced similar ratios of phenotypes Cri:cri of about 19:81 in the TRIS and 27:73 in the TRUST line. An examination of microsporocytes of suspicious slow-developing Cri-plants invariably showed the presence of one small univalent at metaphase I. Pollen fertility of trisomics $(>0.9)$ was as high as in the diploid sibs. The absence of tetrasomics could be explained by the transmission of the extra chromosome mostly through eggs or pollen. However, in testcrosses, carried out for the TRUST line, the extra chromosome was transmitted through about $25 \%$ of eggs and about $12 \%$ of sperm cells (Table 1); hence, we would expect $3 \%$ tetrasomics in the progenies of trisomics.

\section{Synthesis of stable trisomicss}

By treatment of seeds of the TRUST trisomics with ethylmethane sulfonate, we tried to obtain a sporophytic lethal able to bind the extra chromosome to the basic chromosome set. Among the progenies of 207 vigorous trisomic M3 plants, our attention was attracted to two families in which all the plants had the Cri-phenotype and other characters of trisomics. The complete absence of cri-plants could imply that an embryonic recessive lethal had occurred in the H-region. In one family, only about a quarter of seeds gave rise to plants. Later, it was found that in this family the development of the diploid progeny was arrested at the stage of seed germination due to the action of the lethal killer that we mapped to the short arm of chromosome 3 (Berdnikov et al, 2000b). In the other family, all the seeds germinated and gave rise only to trisomics. However, in their pods about one-half 
of embryos ceased development when the seeds were about $2 \mathrm{~mm}$ in diameter. We called this embryonic lethal remover ( $r m v$ ) (Berdnikov et al, 2000b). One vigorous M5 plant was chosen as a founder of a stable trisomic line TRUST-R. For 11 generations of self-pollination, we obtained more than 6000 descendents. All had the phenotypic features of trisomics. An analysis of microsporocytes and root tip cells in several dozens of plants showed the presence of a small additional chromosome (Figure 4 ). The level of pollen fertility ( $>90 \%$ ) of stable trisomics did not differ from that of diploids.

In order to determine the basic chromosome in which the lethal $r m v$ was located, we crossed TRUST-R ( $\mathrm{Crd} A$, cri rmv/Crd A, cri rmv/(Crd, Cri Rmv)) used as a male with the diploid line WHAF (crd $d^{w h} a$, Cri Rmv/ crd $d^{w h} a$, Cri $R m v)$. Note that the genes $a$ and $c r d^{w h}$ are located on chromosome 6 , being separated by 22 crossover units (Berdnikov et al, 2000a). Among 82 plants produced by self-pollinating F1 diploids, only three cri-plants were detected, while the number of plants with the phenotype $c r d^{w h}$ and $a$ were 21 and 27, respectively. The segregation for chromosome 6 markers $c r d^{w h}$ and $a$ corresponded well to the Mendelian 3:1 ratio, but the strong deficit of
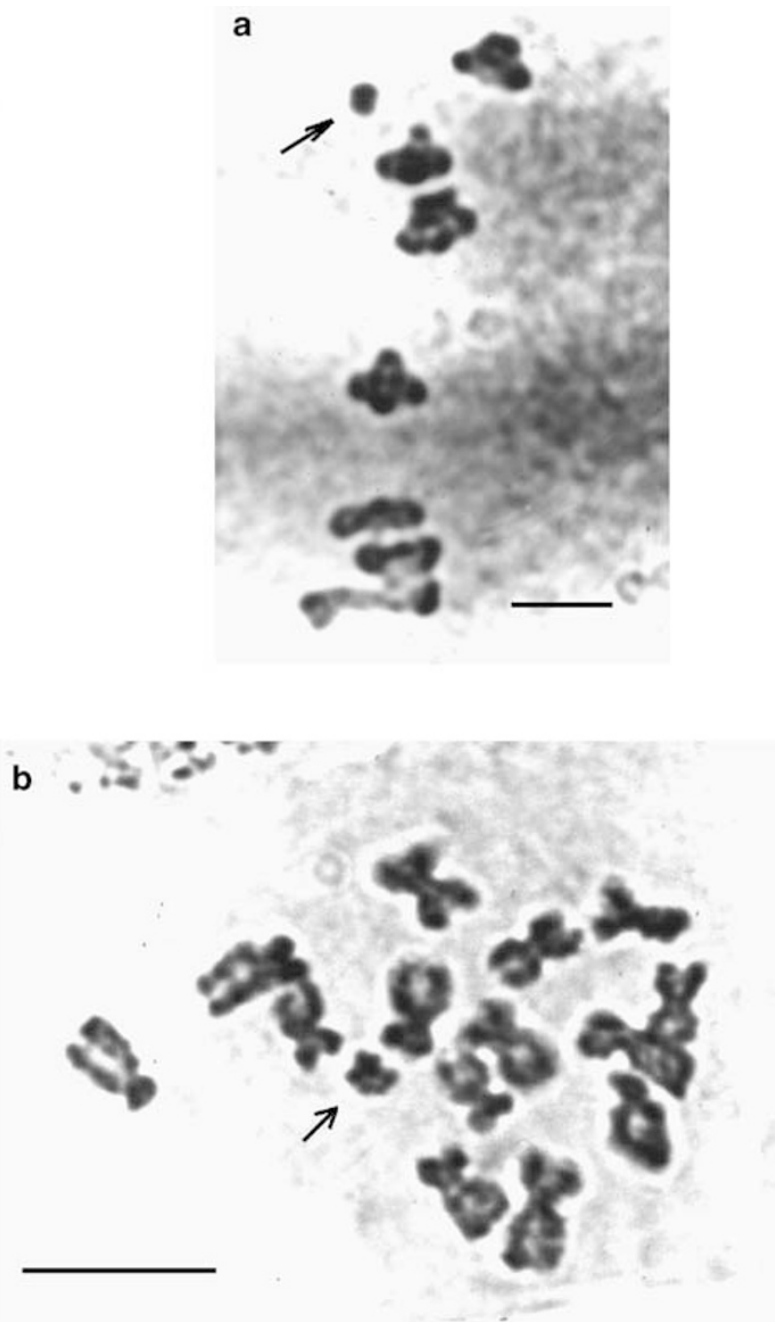

Figure 4 Extra chromosome (indicated by arrowheads) in meiosis and mitosis of the line TRUST-R. (a) metaphase I in pollen mother cells; (b) metaphase in root tip cells. cri-plants pointed to the position of $r m v$ on chromosome $5.6 \pm 1.9 \%(P<0.0001)$. For evaluation of the recombination rate between $r m v$ and cri in trisomics, it was sufficient to estimate the frequency of occurrence of criplants in the line TRUST-R. After scoring more than 2000 plants of this line, we detected no cri-plants. This result confirms the recombinational isolation of the arm of the extra chromosome homologous to the short arm of chromosome 3 .

Reciprocal testcrosses of TRUST-R with the diploid line Yellow Crispa ( $g p$ cri) showed that the transmission of the extra chromosome through eggs and pollen was 34.4 and $17.6 \%$, respectively (Table 1). Based on these values, we should have observed about $13 \%$ of tetrasomics among the progeny of TRUST-R trisomics, but we failed to detect them. It seems likely that the presence of four copies of some gene in the tetrasomic genome is equivalent to a sporophytic lethal. Thus, we have obtained a true breeding line of the garden pea with 15 chromosomes.

In order to test the rate of recombination in both arms of the extra chromosome, we introduced a recessive marker $\mathrm{crd}^{\text {wh }}$ into the short arm of chromosome 6 of a stable trisomic. To do this, we examined the progeny (45 plants) of self-pollinated trisomics produced by the above-described cross TRUST-R $\times$ WHAF $\left(a c r d^{w h}\right)$. Taking into account the fact that the loci $c r d$ and $a$ are linked and $a$ maps to the long arm of chromosome 6 (not covered by the extra chromosome), our attention was attracted to three trisomics with white flowers $(a / a)$. Being selfed, one of them gave rise to the family consisting of trisomics with parental phenotype, that is, all of them had the phenotype $a \mathrm{Cri} \mathrm{Crd}$ and morphological characters of trisomics. One vigorous plant of this family became a founder of another stable trisomic line TRAC. We have scored in total more than 700 individuals of TRAC, but no plants with the phenotypes cri or $c r d^{\text {wh }}$ were detected. At the same time, diploid progenies from crosses of TRAC with diploid testers homozygous for $\mathrm{cri}$ or $c r d^{w h}$ had recessive phenotypes of testers used; hence,

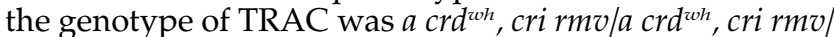
(Crd, Cri Rmv) (Figure 5). Therefore, the absence of $c r d^{w h}$ -

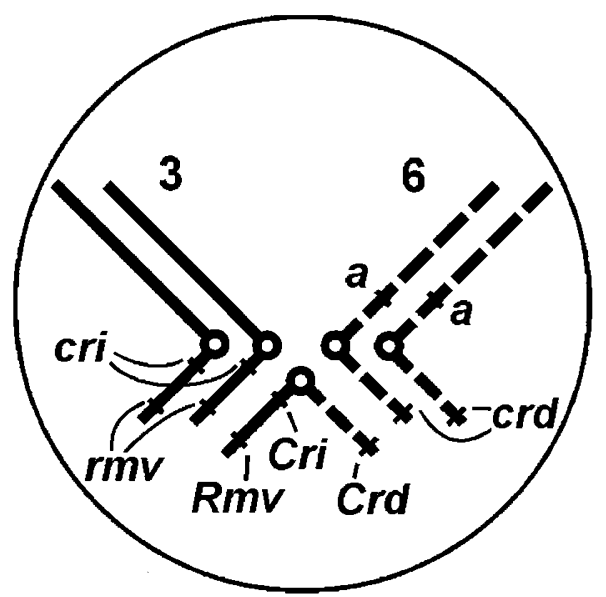

Figure 5 Genetic constitution of trisomics of the line TRAC. Numbers stand for chromosomes 3 and 6 (Linkage Groups V and II). 
plants among TRAC trisomics indicates a strong suppression of recombination in the arm of the extra chromosome homologous to the short arm of chromosome 6 .

\section{Discussion}

The origin of plant B chromosomes from the extra chromosome of tertiary trisomics was first proposed by Tobgy (1943). Jones and Rees (1982) also suggested that future Bs appeared as trisomics or trisomic fragments and then rapidly lost an ability to pair with the homologous region of the genome and switched off their genes in order to overcome genic imbalance. The authors suggested that 'heterochromatinization' was involved in both the transformations. However, a phenomenon similar to $X$ chromosome inactivation in mammalian females is unknown in plants. It seems more likely that the genes of the extra chromosome are deleted or destroyed by mutations. Green (1990) hypothesized that recombinational isolation of an incipient $\mathrm{B}$ chromosome may be caused by a change in the composition of DNA repeats. Although this process is rapid, from an evolutionary point of view, it should take many generations. A question arises as to how the extra chromosome can persist, if it is harmful and can be readily lost at meiosis. Another question is how genes of the extra chromosome can degrade while their alleles on the basic chromosomes remain intact. Of course, the extra chromosome may persist if it is very small (ie, little impairment of genic balance) and, in addition, initially possesses an effective accumulation mechanism or a gene essentially elevating the fitness. However, the probability of occurrence of both these conditions may be extremely low.

To model the initial steps of B chromosome evolution, we have chosen the tertiary trisomic of pea, regularly appearing in the progeny of heterozygotes for Hammarlund T(3-6)a translocation. The karyotype of this trisomic differs from the normal one by addition of a small extra chromosome composed of the short arms of chromosomes 6 and 3. In pea, all the primary and the majority of tertiary trisomics obtained are weak and practically sterile plants (Popova, 1975; Gorel et al, 1992). The tertiary trisomic chosen by us is an exception in being rather vigorous and fertile.

Since a critical requirement for the initial stages of $B$ chromosome evolution is recombinational isolation from the rest of the genome, we tried to estimate the rate of recombination between the extra chromosome and the homologous genomic region (H-region). In the first synthesized trisomic line, TRIS, we found few crossovers between the translocation breakpoint and the closely linked locus cri. However, in the second synthesized trisomic line, TRUST, recombination in this region proved to be completely suppressed. Moreover, crossing-over between the loci cri and $u$ separated by about $40 \mathrm{cM}$ in the map of the short arm of chromosome 3 (Weeden et al, 1998) was suppressed as well. An analysis of microsporocyte metaphase I in TRUST trisomics invariably revealed the extra chromosome as a univalent. The trisomics TRIS and TRUST have the same extra chromosome but differ at least in the distal part of the chromosome 3 short arm with respect to the locus $u$. Taking into account an important role of terminal segments in chromosome pairing (Burnham et al, 1972), replacement of such a segment of the short arm of chromosome 3 may be the cause of the suppression of recombination in the entire extra chromosome. Wiebe et al (1974) described a barley line with an extra pair of short chromosomes presumably derived from the translocation T5-7a. The extra chromosome lost its telomeric regions and failed to pair with the homologous regions in the regular chromosome set (Finch and Kasha, 1976; Fedak, 1977). It seems that the natural intraspecies polymorphism for factors affecting homologous pairing may be sufficient to exclude the short member of a trivalent from recombination. The occurrence of $B$ chromosomes only in cross-pollinating species (Jones and Rees, 1982) may be accounted for by differences in the structure of telomeric regions of homologs in heterozygous individuals.

The problem of retaining a nascent B chromosome in a population during many hundreds of generations could be solved by introducing a sporophytic lethal in the $\mathrm{H}$ region, while the extra chromosome retains the wild-type allele. Gametophytic and sporophytic lethals near translocation breakpoints are shown to be widespread in some plants (Cleland, 1962; James, 1965). However, it is difficult to estimate $a$ priori the likelihood of their arising in a given genome region. Having studied the progenies of only 207 mutagenized plants, we found two recessive sporophytic lethals in the chromosome 3 short arm: remover, which kills the embryo at early developmental stages, and killer, which arrests development at the germination phase (Berdnikov et al, 2000b). Therefore, the origin and survival of a sporophytic mutation in the H-region of a tertiary trisomic seems quite possible, provided that recombination in the extra chromosome is suppressed. With the aid of the lethal $r m v$, we obtained two stable trisomic lines - TRUST-R with visible markers in the short arm of chromosome 3 and TRAC with markers in short arms of chromosomes 3 and 6. Surprisingly, we failed to detect tetrasomics in the progenies of our stable trisomics. The expected proportion of tetrasomics in the progenies of self-pollinated trisomics of the line TRUST-R was about 13\%. Their absence suggests that tetrasomics die at early developmental stages, presumably due to severe genic imbalance.

Thus, we obtained stable pea lines with 15 chromosomes, wherein the extra chromosome is always present as a single element with no partners for pairing. If such trisomics occurred in nature, their extra chromosomes may develop into B chromosomes. We propose that natural selection at the gametophyte level will result in the preferential impairment of genes of the extra chromosome, since loss-of-function mutations are neutral or slightly advantageous for $n+1$ gametophytes, if they occur in the extra chromosome, and are harmful for haploid gametophytes, if such mutations occur in the $\mathrm{H}$ region. This process, taking into account the substantial overlap of genes operating at the haplo- and diplophases of the angiosperm life cycle (Mulcahy, 1979; Mascarenhas, 1989; Ottaviano and Mulcahy, 1989), will lead to inactivation of the majority of extra chromosome genes.

Below, we present our view of the main stages of $B$ chromosome evolution from the extra chromosomes of tertiary trisomics. We consider all events to take place in populations of plants propagating both through selfand cross-pollination. It is assumed that individuals 
heterozygous for a reciprocal translocation occur with noticeable frequencies and the population size may vary greatly from year to year.

\section{The first stage}

In a heterozygote for translocation, a sporophytic lethal occurs in a chromosome region near the translocation breakpoint. Self-pollination of such a plant will give the progeny containing tertiary trisomics in which two copies of the lethal gene are covered by the wild-type allele in the extra chromosome.

\section{The second stage}

At a moment when the population size is reduced to solitary plants, a population of stable trisomics can be founded.

\section{The third stage}

If the population of stable trisomics is well isolated from diploid populations of the same species, it has time for independent evolution. During this time, if crossing-over in the extra chromosome is suppressed, natural selection, acting predominantly at the gametophyte level, will accumulate deletions and loss-of-function mutations in the extra chromosome. Eventually, the fitness of trisomics reaches that of diploids. Additionally, an accumulation mechanism increasing the proportion of $n+1$ gametes may be developed.

\section{The fourth stage}

After a period of isolation, the population of former stable trisomics can contact a population of diploids of the same species. If the supernumerary chromosome devoid of most functional genes had succeeded in the acquisition of an accumulation mechanism, it can persist in a new genome as well. At this final stage, the neutral mechanism of Muller's ratchet, that is, stochastic loss of the least degraded extra chromosomes (Green, 1990), can induce inactivation of the remaining genes residing in the extra chromosome, turning it into a true B chromosome.

\section{Acknowledgements}

This work was supported by the Grant no 99-04-49970 of the Russian Foundation for Fundamental Research (RFFI) and the Grant no 106/43-5 of the Ministry of Industry, Technology and Science of Russian Federation.

\section{References}

Adams MD, Celniker SE, Holt RA, Evans CA, Gocayne JD, Amantides PG et al (2000). The genome sequence of Drosophila melanogaster. Science 287: 2185-2195.

Ashburner M (1989). Drosophila. A Laboratory Handbook. Cold Spring Harbor Laboratory Press: Cold Spring Harbor. pp 691-716.

Berdnikov VA, Gorel FL, Kosterin OE (2000a). A new allele at $\mathrm{Crd}$ disturbs development of the compound leaf. Pisum Genet 32: 6-8.

Berdnikov VA, Gorel FL, Kosterin OE (2000b). New mutations in the linkage group $\mathrm{V}$ have provided stable trisomic lines of pea. Pisum Genet 32: 13-15.

Berdnikov VA, Gorel FL, Temnykh SV (1993). A more precise location of the breakpoints in Hammarlund's K-line. Pisum Genet 25: 18-21.
Bukeboom LW (1994). Bewildering Bs: an impression of the 1st B-chromosome conference. Heredity 73: 328-336.

Burnham CR, Stout JT, Weinheimer WH, Kowles RV, Phillips RL (1972). Chromosome pairing in maize. Genetics 71: 111-126.

Cleland RE (1962). The cytogenetics of Oenothera. Adv Genet 11: 147-237.

Colombo PC, Remis MI (1997). On the origin of B-chromosomes: neo $\mathrm{XY}$ systems and X-like supernumeraries in Orthoptera. Caryologia 50: 151-162.

Fedak G (1977). Meiosis in 8-chromosome barley haploids. Can J Genet Cytol 19: 211-216.

Finch RA, Kasha KJ (1976). Meiotic associations in barley lines with eight pairs of chromosomes and their haploids. Can J Genet Cytol 18: 753-761.

Gorel FL, Temnykh SV, Lebedeva IP, Berdnikov VA (1992). Twisted tendrils (Twt) - a phenotype associated with a translocation involving chromosome 1. Pisum Genet 24: 48-51.

Green DM (1990). Muller's ratchet and the evolution of supernumerary chromosomes. Genome 33: 818-824.

Green DM, Zeyl CW, Sharbel TF (1993). The evolution of hypervariable sex and supernumerary chromosomes in the relict New Zealand frog Leiopelma hochstetteri. J Evol Biol 6: 417-441.

James SH (1965). Complex hybridity in Isotoma petraea I. The occurrence of interchange heterozygosity, autogamy and a balanced lethal system. Heredity 20: 185-197.

Jones RN, Rees H (1982). B Chromosomes. Academic Press: New York.

Khush GS, Rick CM (1967). Tomato tertiary trisomics: origin, identification, morphology, and use in determining position of centromeres and arm location of markers. Can J Genet Cytol 9: 610-631.

Kosterin OE, Berdnikov VA, Gorel FL (1999). Equations for estimation of recombination fraction between a marker and a sporophytic lethal in F2 progenies with the maximum likelihood method. Pisum Genet 31: 33.

Lamm R (1986). A new interpretation of Hammarlund's K-line. Pisum Newslett 18: 34-36.

Lewis H (1951). The origin of supernumerary chromosomes in natural populations of Clarkia elegans. Evolution 5: 142-157.

Mascarenhas JP (1989). The male gametophyte of flowering plants. Plant Cell 1: 657-664.

Mulcahy DL (1979). The rise of the angiosperms: a genecological factor. Science 206: 20-23.

Ottaviano E, Mulcahy DL (1989). Genetics of angiosperm pollen. Adv Genet 26: 1-64.

Pellew C (1940). Genetical studies on the first reciprocal translocation found in Pisum sativum. J Genet 39: 363-390.

Popova IS (1975). Cytologic studies. In: Khvostova VV (ed) Genetics and Selection of Pea. Nauka: Novosibirsk. pp 106-141 (in Russian)

Ramage RT (1960). Trisomics from interchange heterozygotes in barley. Agron J 52: 156-159.

Sutton E (1939). Trisomics in Pisum sativum derived from an interchange heterozygote. I Genet 38: 459-476.

Tobgy HA (1943). A cytological study of Crepis fuliginosa, C. neglecta and their F1 hybrid and its bearing on the mechanism of phytogenic reduction in chromosome number. J Genet 45: 67-111.

Vasek FC (1961). Trisomic transmission in Clarkia unguiculata. Am J Bot 48: 829-833.

Wedberg HL, Lewes H, Venkatesh CS (1968). Translocation heterozygotes and supernumerary chromosomes in wild populations of Clarkia williamsonii. Evolution 22: 93-107.

Weeden NF, Ellis THN, Timmerman-Vaughan GM, Swiecicki WK, Rozov SM, Berdnikov VA (1998). A consensus linkage map for Pisum sativum. Pisum Genet 30: 1-4.

Wiebe GA, Ramage RT, Eslick RF (1974). Eight-paired barley lines. Barley Genet Newslett 4: 93-95. 